\title{
Advances in adolescent research in the Chinese culture: reflections and future research directions
}

\section{Daniel T.L. Shek, Rachel C.F. Sun and Joav Merrick}

A survey of the literature showed that there are worrying trends and phenomena related to the development of adolescents in different parts of the world, such as mental health problems, abuse of psychotropic substances, suicide and unemployment problems. In view of these growing adolescent developmental issues, there is a need to understand the nature of these problems so that appropriate preventive measures can be devised. Unfortunately, advance in research on adolescence is much slower than the growth of adolescent problems. For example, in a discussion of quality of life research in different stages of the lifespan (1), it was observed that although much research has been conducted to examine the quality of life of people in different stages of the lifespan, such as adulthood in general and old age in particular, there are comparatively fewer research studies on the quality of life of children and adolescents.

In addition, there are several deficits in the existing adolescent research studies. The first deficit concerns how adolescent behavior is explained and understood. Although there are many studies examining adolescent behavior from a psychological perspective (i.e., micro-view) or sociological perspective (i.e., macro-view), there is a growing consensus among social scientists and allied professionals maintaining that human behavior is embedded in different ecological systems (e.g., family system and macro socio-cultural system). As such, it is obvious that a more holistic understanding of adolescent behavior and the related phenomena with reference to different systems is called for (2). With reference to different micro- and macro-systems, the family system can be regarded as an important developmental context, particularly in the Asian culture. Unfortunately, studies on family quality of life with reference to adolescents are sparse (1). There is also growing concern about how families should be studied as families commonly involve different members which are more than aggregates of individuals. Methodologically speaking, studies on families can require different methodologies (such as capturing the views of different dyads) and statistical analyses (such as hierarchical linear modeling).

Second, as pointed out earlier (1), comparatively fewer studies have examined quality of life of adolescents experiencing economic disadvantage. Poverty has been a growing global problem in the past decades. In Third world countries, poverty is a prime social problem to be addressed. Even in developed countries, poverty has not diminished in proportion to economic growth. For example, it is estimated that one out of four children in America live in poverty and $40 \%$ of poor people in the United States are children. Because poverty is a rising global problem, it is important to understand how people explain the causes of poverty and the impact of poverty on adolescents. Unfortunately, in contrast to the growing problem of poverty in the global context, studies of poor families have not received the same level of research focus as families in the middle class. Obviously, more studies on adolescents experiencing economic disadvantage in the contexts of families, communities and societies should be conducted.

Third, although there are numerous research studies on the relationships between adolescents and their significant-others, such as parents and peers, there are comparatively fewer studies examining how adolescents interact with old people, and the issue of intergenerational solidarity is much underresearched. In particular, there is a paucity of research studies on how adolescents perceive old people in the Chinese culture. Obviously, with the growing aging population in the global context, it is important to understand how adolescents perceive and interact with old people.

The fourth limitation of the existing scientific literature is that the majority of the studies have been conducted in Western contexts based on Western participants and comparatively fewer studies have been conducted in different Chinese contexts $(3,4)$. For example, with reference to PsycINFO, although there were 75,086 citations when the term "parenting" was used, similar search using "parenting" and "Chinese" showed that there were only 5,522 citations in September 2010. Obviously, this is not a problem if the assumption that parenting and the related phenomena are universal in different cultures is valid. However, because culture plays an important role in the definition and experience of parenting, the predominance of studies based on non-Chinese participants is an obvious limitation $(5,6)$. Furthermore, there are few studies examining the cross-cultural applicability of family and parenting constructs which are mainly developed in the West. As such, it is important to conduct more studies in Chinese cultural contexts to understand cross-cultural differences in adolescent development. In particular, with reference to the question of generalizability of parenting theories and research findings generated from the Western contexts, studies in different Chinese contexts are indispensable.

There are several reasons why more attention should be placed on research based on Chinese adolescents. First, the size of the Chinese population warrants research attention. The World Population Clock showed that the population of China was $1,330,141,295$ in 2010, which is approximately one-fifth of the population of the world in 2010 . The 
implication of these figures is that if any theory is claimed to be universally applicable, relevant data from Chinese people must be collected. Second, China has a history of over 5,000 years and family has been strongly emphasized in the Chinese culture. Cultural beliefs in Chinese culture provide food for thought for researchers on adolescence in non-Western cultures. Finally, with the establishment of a Socialist political system in 1949, development of the Cultural Revolution in the mid-1960s to mid-1970s, and adoption of the open door policy and economic reforms in China since late 1970s, it is important to examine how Chinese adolescents and their families have been transformed in the past decades.

Another deficit in the literature on adolescent research concerns substance abuse. Probably because of the influence of the popular culture and youth subculture, substance abuse among young people has become an acute global problem. Smoking, alcohol abuse, and use of illicit drugs such as ecstasy and ketamine are growing concerns. As such, many studies have been conducted to examine the risk and protective factors and preventive drug education (2). Nevertheless, comparatively fewer studies have been conducted to examine treatment of young substance abusers. Scientific studies on treatment among young substance abusers are important because there is a need to better engage young substance abusers in the treatment process (7). In addition, we know very little about treatment fears among young substance abusers and such understanding is important as far as evidencebased and effective treatment is concerned. As such, there is a need to understand the help-seeking process and treatment experiences of adolescent substance abusers.

Finally, with the changing landscape in the paradigms of social science research, positivistic and post-positivistic research studies no longer monopolize adolescent research, and studies adopting interpretive and constructionist approaches become popular (8). Unfortunately, there are few useful references in the field of adolescent medicine and health addressing the similarities and differences of the two approaches.

Against the above background, the papers in this special issue attempt to address the missing gaps in adolescent development, medicine and health. The topics of adolescent issues in this special issue include conceptual gaps and methodological issues in the studying of parenting in Hong Kong, conceptual and methodological issues in the studying of adolescents experiencing economic disadvantage, intergenerational solidarity and perceptions of old people among adolescents in
Hong Kong, and treatment fears among young substance abusers in Hong Kong. In addition, one conceptual paper and one empirical paper on the application of interpretive and social constructionist principles in adolescent research are presented. We earnestly hope that these papers can constitute food for thought for colleagues not just in different Chinese communities but also in other parts of the world.

\section{References}

1. Shek DT. Quality of life of Chinese people in a changing world. Soc Indic Res 2010;95:357-61.

2. Shek DT. Tackling adolescent substance abuse in Hong Kong: where we should go and should not go. ScientificWorldJournal 2007; 7:2021-30.

3. Shek DT, Sun RC. Effectiveness of the Tier 1 Program of Project P.A.T.H.S.: findings based on three years of program implementation. ScientificWorldJournal 2010;10:1509-19.

4. Shek DT, Sun RC. Development, implementation and evaluation of a holistic positive youth development program: project P.A.T.H.S. in Hong Kong. Int J Disabil Hum Dev 2009;8:107-17.

5. Sun RC, Shek DT. Life satisfaction, positive youth development, and problem behavior among Chinese adolescents in Hong Kong. Soc Indic Res 2010;95:455-74

6. Shek DT, Merrick J. Promoting positive development in Chinese adolescents: the project P.A.T.H.S. in Hong Kong. Int Public Health J 2009;1:237-41.

7. Shek DT. School drug testing: a critical review of the literature. ScientificWorldJournal 2010;10:356-65.

8. Lit SW, Shek DT. Application of social constructionist principles in field practice teaching in a Chinese context. Soc Work Educ 2007;26:359-75.

Daniel T.L. Shek, Chair Professor, PhD, FHKPS, BBS, JP, Department of Applied Social Sciences, The Hong Kong Polytechnic University, Hunghom, Hong Kong, P.R. China E-mail: daniel.shek@polyu.edu.hk

Rachel C.F. Sun, Assistant Professor, $\mathrm{PhD}$,

Faculty of Education, The University of Hong Kong, Pokfulam Road, Hong Kong, P.R. China

E-mail: rachels@hku.hk

Joav Merrick, Professor, MD, MMedSci, DMSc, National Institute of Child Health and Human Development, Division for Mental Retardation, Ministry of Social Affairs, Jerusalem, Israel

E-mail: jmerrick@zahav.net.il 